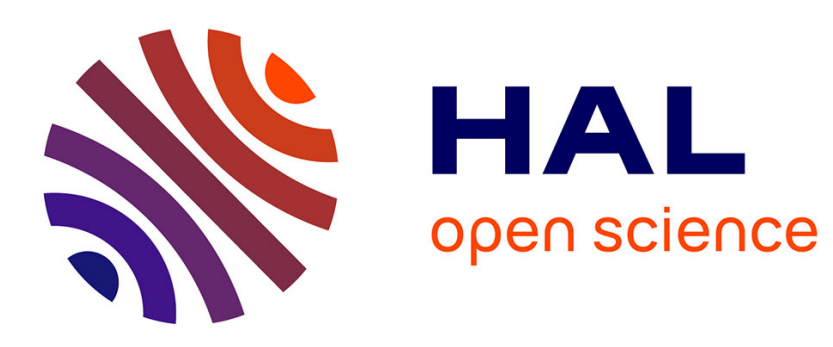

\title{
Active estimation of the initial phase for brushless synchronous motors
}

Jérémy Malaizé, Jean Lévine

\section{To cite this version:}

Jérémy Malaizé, Jean Lévine. Active estimation of the initial phase for brushless synchronous motors. 9th IEEE International Workshop on Advanced Motion Control, May 2007, Istanbul, Turkey. pp.563, 10.1109/AMC.2006.1631721 . hal-00575660

\section{HAL Id: hal-00575660}

https://hal-mines-paristech.archives-ouvertes.fr/hal-00575660

Submitted on 10 Mar 2011

HAL is a multi-disciplinary open access archive for the deposit and dissemination of scientific research documents, whether they are published or not. The documents may come from teaching and research institutions in France or abroad, or from public or private research centers.
L'archive ouverte pluridisciplinaire HAL, est destinée au dépôt et à la diffusion de documents scientifiques de niveau recherche, publiés ou non, émanant des établissements d'enseignement et de recherche français ou étrangers, des laboratoires publics ou privés. 


\section{Active Estimation of the Initial Phase for Brushless Synchronous Motors}

\author{
Jérémy Malaizé ${ }^{1,2}$ \\ ${ }^{1}$ MICRO-CONTROLE Spectra-Physics S.A \\ Z.I. Beaune-la-Rolande, Beaune-la-Rolande, 45340, France \\ jeremy.malaize@newport-fr.com
}

\author{
Jean Lévine ${ }^{2}$ \\ ${ }^{2}$ Centre Automatique et Systèmes \\ École Nationale Supérieure des Mines de Paris \\ 35 rue Saint-Honoré, Fontainebleau, 77300, France \\ jean.levine@ensmp.fr
}

\begin{abstract}
This paper addresses the initial phase estimation problem for brushless synchronous motors. Only displacement measurements are used (no current) and friction, load and motor parameters are supposed to be unknown. Because of friction, the system is modelled by a differential equation with discontinuous right-hand side. Specific open-loop inputs are designed (active method) to get the initial phase as a function of the magnitude of the displacements along the corresponding trajectories. The estimation relies on a complete classification of the possible dynamical behaviours of the considered discontinuous right-hand side system with periodic input, whatever values the unknown parameters may take. We propose an approximated formula of the initial phase. Some experimental results are given, together with a comparison of our method to a classical procedure.
\end{abstract}

\section{INTRODUCTION}

We consider both rotary and linear synchronous motors used in high precision positioning applications, such as the semiconductor wafer industry. Brushless motors are preferred to DC motors with brushs for their longer life time, improved cleanliness of operation and better resistance to wear and tear. For DC motors (with brushs), current commutations, as well as initialization, are performed mechanically while, for brushless motors (without mechanical contact), additionnal measurements and an algorithm are required for the currents and the magnetic field to be in phase. The initialization procedure aims at getting the currents in phase with the position of the motor coils inside the magnetic field. In this paper, we propose a method using only displacement measurements to determine the initial rotor position (modulo the magnetic pitch) for brushless synchronous motors ${ }^{1}$.

When it comes to reducing the number of sensors for economical reasons, current sensors are usually preferred to position encoders. Several papers address sensorless control of synchronous motors, that is to say the determination of the initial rotor position from current measurements. Some existing techniques consist in using an observer to estimate the back-electromotive force induced in the coils, see [4] and [8] for example. Other approaches determine the motor windings inductance by injecting either specific carrier-frequency signals ([11], [12] or [9]) or no signal at all ([7] or [10]). Both these techniques rely on current measurements to determine an electrical variable in phase with the magnetic field.

\footnotetext{
${ }^{1}$ US patent pending.
}

For the aforementionned applications featuring nanometric positioning precision, high resolution position sensors turn out to be necessary. Optical resolvers, or absolute position sensors, are too expensive and, in this paper, only optical incremental position sensors measuring the relative displacement around the initial position are considered. We propose a method that provides an autonomous current controller (whose current measurements are not made available to the initialization algorithm) with specific inputs computed offline and simply gets the corresponding displacement measurements back to estimate the initial rotor position. This estimation scheme, illustrated figure 1, spares the use of additional and unnecessary Hall effect sensors.

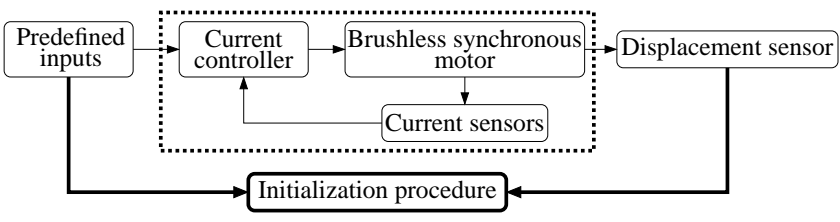

Fig. 1. Estimation Scheme.

An existing solution ${ }^{2}$, falling under the scope of the previously defined scheme, consists in maintaining a constant current in an electrical phase until the mechanical equilibrium. The corresponding position shift is then used to compute an estimate of the initial phase. Unfortunately, before stabilizing, erratic oscillations around the equilibrium position are witnessed. These movements are as large as the magnetic pitch (typically a couple of millimeters) and cannot be avoided. This behaviour might not be suitable for the aforementionned high precision applications.

In this paper, the method can be tuned to generate arbitrarily small magnitude displacements (a couple of microns). According to scheme 1, neither magnetic field nor currents have to be fed to the algorithm and the motor parameters are unknown (gain, load and friction). In these conditions, our method features an accurate initial phase estimation with little computation, which makes real-time implementation easier.

This paper is organized as follows. We first model the dynamics of the brushless synchronous motors and highlight the need for a precise initial position estimation : guaranteeing

\footnotetext{
${ }^{2}$ Hereafter referred to as the classical method.
} 


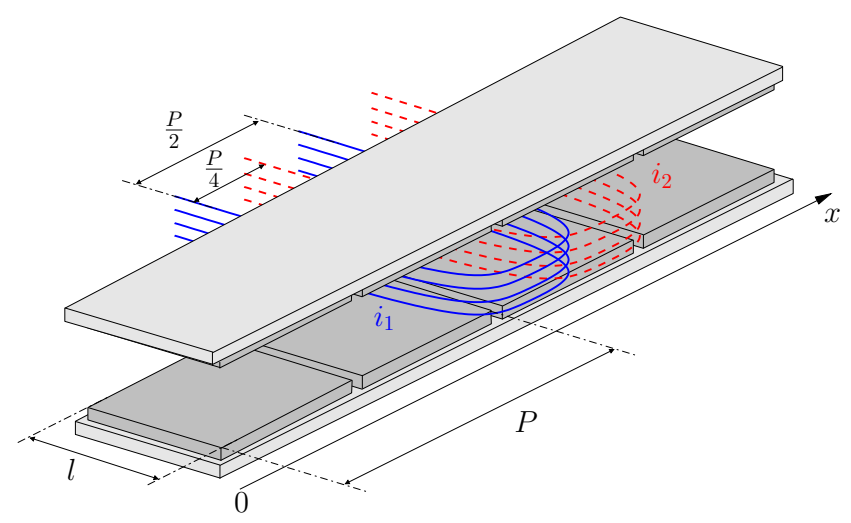

Fig. 2. Two-phase ironless linear motor.

stable and accurate positioning together with an optimal use of the motor, from an energetic point of view (section II). We then derive the relation between the initial phase, the other unknown parameters and the magnitude of the displacements when the system is driven by a periodic and open-loop input (section III). This analysis is based on a complete classification of the periodic orbits of the considered system under forced oscillations. In particular, it is shown how the friction, modelled by a function of the sign of the speed (thus giving rise to a discontinuous right-hand side system in the sense of Filippov (see [5])), affects the trajectories followed by the system. Then, we compare the measured displacements to those predicted by the classification, and, thanks to an approximation, the initial position is obtained. In section IV, experimental results are provided to consolidate the different assumptions and show that our initialization procedure outperforms the classical method.

\section{INITIAL PHASE ESTIMATION}

\section{A. Modelling}

For simplicity's sake, only two-phase linear motors (depicted on figure 2) are allowed for since the generalization is rather obvious. Referring to figure 2, a track made up of permanent magnets creates a sinusoidal magnetic field whose magnitude and spatial period are respectively noted $B_{0}$ (unknown) and $P$ (given). Let $l$ be the length of the active windings so that a force $F_{1}$ is created by the electrical phase $\# 1$ :

$$
\begin{aligned}
F_{1} & =i_{1} l B_{0} \sin \left(\frac{2 \pi}{P} x\right)+\left(-i_{1}\right) l B_{0} \sin \left(\frac{2 \pi}{P}\left(x-\frac{P}{2}\right)\right) \\
& =K_{m} i_{1} \sin \left(\frac{2 \pi}{P} x\right) \\
& =K_{m} i_{1} \sin \left(\frac{2 \pi}{P}\left(x_{0}+d\right)\right),
\end{aligned}
$$

with $K_{m}=2 l B_{0}$ the motor gain, $x_{0}$ the initial position and $d$ the measured displacement. By calculations similar to (1), the force created by the second electrical phase is derived :

$$
F_{2}=K_{m} i_{2} \cos \left(\frac{2 \pi}{P}\left(x_{0}+d\right)\right) .
$$

Let's assume a controller stabilizes the currents $i_{1}$ and $i_{2}$ so that their dynamics turn out to be fast and negligible compared to the mechanical behaviour of the system. The whole system is thus modelled by :

$$
\begin{aligned}
\ddot{x}= & \frac{K_{m}}{m} i_{1} \sin \left(\frac{2 \pi}{P}\left(x_{0}+d\right)\right)+\frac{K_{m}}{m} i_{2} \cos \left(\frac{2 \pi}{P}\left(x_{0}+d\right)\right) \\
& -\frac{f}{m} \operatorname{sign}(\dot{x}) .
\end{aligned}
$$

The friction force $f$, the gain $K_{m}$ and the load $m$ are unknown.

To drive (3) when $x_{0}$ is unknown, let's assume $\widehat{x}_{0}$, an estimate of $x_{0}$, is available (keep in mind the determination of $\widehat{x}_{0}$ motivates this paper) and make the following choice for the currents $i_{1}$ and $i_{2}$ :

$$
\begin{aligned}
& i_{1}=I \sin \left(\frac{2 \pi}{P}\left(\widehat{x}_{0}+d\right)+\varphi\right) \\
& i_{2}=I \cos \left(\frac{2 \pi}{P}\left(\widehat{x}_{0}+d\right)+\varphi\right),
\end{aligned}
$$

with $I$ a current reference and $\varphi$ a supplementary degree of freedom. Both $I$ and $\varphi$ may be time-varying functions or not.

Equation (3) together with the choice (4) finally writes :

$$
\ddot{x}=\frac{K_{m}}{m} \cos \left(\varphi_{0}-\widehat{\varphi}_{0}-\varphi\right) I-\frac{f}{m} \operatorname{sign}(\dot{x}),
$$

with $\varphi_{0}=\frac{2 \pi}{P} x_{0}$ the initial phase and $\widehat{\varphi}_{0}=\frac{2 \pi}{P} \widehat{x}_{0}$.

Let $\widehat{K}_{m}$ and $\widehat{m}$ be a priori estimates of $K_{m}$ and $m$, respectively. Since $\frac{\widehat{K}_{m}}{\widehat{m}} I$ is homogenous to an acceleration, from now on, let's note :

$$
\frac{\widehat{K}_{m}}{\widehat{m}} I=\ddot{x}_{r e f},
$$

which, together with $\alpha=\left(\frac{K_{m}}{m}\right) \times\left(\frac{\widehat{m}}{\widehat{K}_{m}}\right)$, leads to the following model of the system :

$$
\ddot{x}=\alpha \cos \left(\varphi_{0}-\widehat{\varphi}_{0}-\varphi\right) \ddot{x}_{r e f}-\frac{f}{m} \operatorname{sign}(\dot{x}) .
$$

\section{B. Role of the initial phase}

From now on, our concern is to get an estimate of the initial phase from (6) by appropriately choosing $\ddot{x}_{r e f}$ and $\varphi$ and despite the lack of knowledge on the other parameters.

Let $\varphi=0$, then the force created by the motor is :

$$
F=F_{1}+F_{2}=K_{m} \cos \left(\varphi_{0}-\widehat{\varphi}_{0}\right) I,
$$

and, consequently :

$$
|F| \leq\left|K_{m} I\right|=m \alpha\left|\ddot{x}_{r e f}\right| .
$$

Thus, for a given acceleration reference $\ddot{x}_{r e f}$, as soon as the relation $\widehat{\varphi}_{0} \neq \varphi_{0}$ holds, the norm of the force is lower than the desired value. The energetic transfer between the current and the force is optimal when $\widehat{\varphi}_{0}$ is accurately determined. Moreover, the gain of the open loop from $x$ to $\ddot{x}_{r e f}$ depends on $\cos \left(\varphi_{0}-\widehat{\varphi}_{0}\right)$. Therefore, the accuracy of the estimation is also material to guarantee both the precision and the stability of the positioning. 


\section{ESTIMATION PROCEDURE}

\section{A. Choice of the trajectories}

We now propose a specific input $\ddot{x}_{r e f}$ that both generates displacements as small as possible and differentiates between the contributions of the different unknown parameters of the model (6).

Note that, under the following assumptions :

$$
\alpha=1, \quad \widehat{\varphi}_{0}=0, \quad f=0,
$$

if $\ddot{x}_{r e f}$ is the second derivative of an oscillating trajectory of magnitude $\xi_{1}-\xi_{0}$, the magnitude of the measured displacements is immediately given by $\cos \left(\varphi_{0}-\varphi\right)\left(\xi_{1}-\xi_{0}\right)$. Choosing $\varphi=0$ and $\varphi_{0}=\frac{\pi}{2}$ successively gives an estimation of $\cos \left(\varphi_{0}\right)$ and $\sin \left(\varphi_{0}\right)$. This way, the small magnitude constraint is fulfilled by appropriately tuning $\xi_{1}-\xi_{0}$ and the initial phase is easily obtained.

Let's design such an oscillating trajectory by first defining an elementary trajectory from an initial point $\xi_{0}$, at rest, to a final point $\xi_{1}$, also at rest, in a time $T>0$ :

1) Let's first impose the following initial conditions :

$$
x_{\text {elem }}(0)=\xi_{0}, \quad \dot{x}_{\text {elem }}(0)=0, \quad \ddot{x}_{\text {elem }}(0)=0 .
$$

2) Similarly, the final conditions are :

$$
x_{\text {elem }}(T)=\xi_{1}, \quad \dot{x}_{\text {elem }}(T)=0, \quad \ddot{x}_{\text {elem }}(T)=0 .
$$

3) A possible choice for $x_{\text {elem }}(t)$ is the polynomial interpolation of degree 5 given by :

$$
x_{\text {elem }}(t)=\xi_{0}+\left(\xi_{1}-\xi_{0}\right) \sum_{i=1}^{5} a_{i}\left(\frac{t}{T}\right)^{i} .
$$

4) The coefficients $\left\{a_{i}\right\}_{1<i<5}$ are derived by identifying (7) at $t=0$ and $t=T$ with the initial and final conditions, to eventually get :

$$
a_{1}=a_{2}=0, \quad a_{3}=10, \quad a_{4}=-15, \quad a_{5}=6 .
$$

The reverse trajectory from $\xi_{1}$, at equilibrium, to $\xi_{0}$, at equilibirum, in a time $T>0$ is similarly derived. Finally, let $x_{M}$ be the trajectory made up of $M$ round trips from $\xi_{0}$ to $\xi_{1}$ and back to $\xi_{0}$, using interpolation (7).

Because of the unknown parameters in (6), when $\ddot{x}_{r e f}=\ddot{x}_{M}$, the system does not follow the trajectory $x_{M}$ (keep in mind this is an open-loop identification procedure) unless the following conditions are fullfilled :

$$
\alpha=1, \quad \widehat{\varphi}=\varphi_{0}, \quad \varphi=0, \quad f=0,
$$

that is to say when all the parameters are perfectly known. As long as $f=0$, only the magnitude of the displacements is affected by the unknown parameters and the measured trajectory is proportional to $x_{M}$. On the other hand, when $f \neq 0$, the friction introduces a delay.

This brief study suggests that, when $\ddot{x}_{r e f}=\ddot{x}_{M}$, it is possible to ditsinguish between the different unknown parameters as they delay or rescale the reference trajectory $x_{M}$. We now propose a complete analysis for in-depth explanation of how to extract the initial phase from the measured displacements despite unknown parameters.

\section{B. Dynamical behaviours classification}

The idea underlying the proposed method is to integrate equation (6) twice, with $\ddot{x}_{r e f}=\ddot{x}_{M}$, to explicit the relation between the measured displacements around the initial position and the different parameters of the model featuring a discontinuous right-hand side. As addressed in [5], in the vicinity of the discontinuity surface, whether the system crosses the surface or slides on it directly depends on the model parameters. This link is pointed out hereafter by introducing the reduced parameter $\mu$, whose definition is given by :

$$
\mu=\alpha m\left|\cos \left(\varphi_{0}-\widehat{\varphi}_{0}-\varphi\right)\right| \ddot{x}_{\max } / f,
$$

with $\ddot{x}_{\max }$ the maximal value of the reference acceleration $\ddot{x}_{M}$ :

$$
\ddot{x}_{\max }=\max _{0 \leq t \leq T}\left|\ddot{x}_{\text {elem }}(t)\right| .
$$

As stated in theorem 1 , the value $\mu$ leads to a complete classification of the dynamical behaviours of the system (6) driven by $\ddot{x}_{M}$. This theorem makes up an essential prerequisite to integrate (6) twice.

Theorem 1: For each $\mu>1$, there exists only one attractive periodic orbit for the system (6) initially at rest with $\ddot{x}_{r e f}=\ddot{x}_{M}$.

There also exist two real numbers $\mu_{1}$ and $\mu_{2}, 1<\mu_{1} \leq \mu_{2}$, depending only on $\ddot{x}_{M}$, that define three and only three possible behaviours :

(i) $1<\mu \leq \mu_{1} \approx 1.4$ : the system instantaneously reaches a periodic orbit along which sliding on the surface of discontinuity $\dot{x}=0$ occurs (see simulation results ${ }^{3}$ figure $3)$.

(ii) $\mu_{1}<\mu \leq \mu_{2} \approx 1.7$ : the system reaches a periodic orbit in finite time (lower than $2 T$ ) along which sliding on the surface of discontinuity $\dot{x}=0$ occurs (see simulation results figure 4).

(iii) $\mu>\mu_{2}$ : the system asymptotically reaches a periodic orbit and no sliding occurs along this orbit (see simulation results figure 5).

Moreover, if $\mu \leq 1$, the system remains at rest.
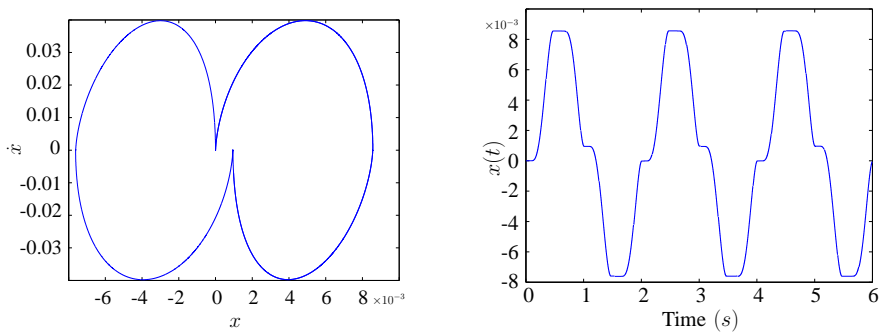

Fig. 3. State space (left) and temporal (right) trajectories for $1<\mu \leq \mu_{1}$.

\footnotetext{
${ }^{3}$ The following reduced system is simulated : $\ddot{x}=\frac{\ddot{x}_{M}}{\ddot{x}_{\max }}-\mu^{-1} \operatorname{sign}(\dot{x})$.
} 

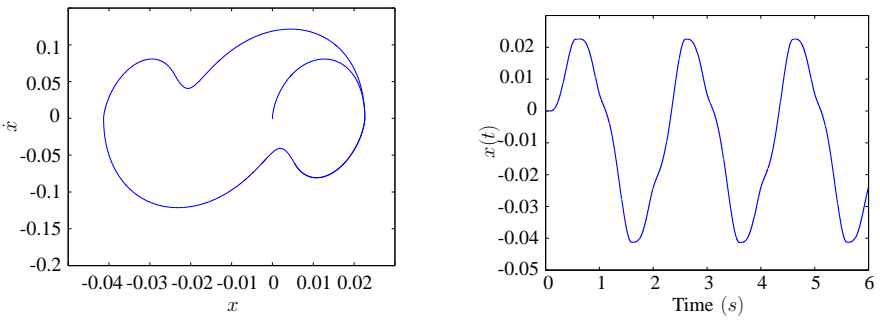

Fig. 4. State space (left) and temporal (right) trajectories for $\mu_{1}<\mu \leq \mu_{2}$.
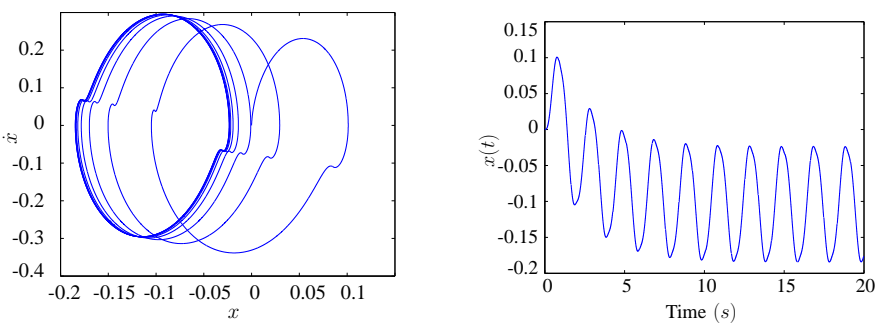

Fig. 5. State space (left) and temporal (right) trajectories for $\mu>\mu_{2}$.

\section{Integration of (6)}

According to theorem 1, whatever value $\mu$ may take, the system reaches a periodic orbit for sure and it is possible to distinguish between orbits sliding on the surface of discontinuity $\left(\mu \leq \mu_{2}\right)$ or not $\left(\mu>\mu_{2}\right)$. This classification makes the integration of (6) tremendously easier and let $\delta$ be the magnitude of the displacements on the orbit :

$$
\delta=\lim _{k \rightarrow \infty}\left(\max _{0 \leq \tau \leq T}|x(k T+\tau)-x(k T)|\right) .
$$

$\delta$ is naturally related to $\mu$ and thus to the initial phase. The theorem 2, that, for simplicity reasons, we give without proving it, states this relation :

Theorem 2: For the system (6) with $\ddot{x}_{r e f}=\ddot{x}_{M}$, there exists a function $\Delta$ defined on $\mathbb{R}^{+}$and taking values in $[0,1[$ so that :

$$
\delta=\alpha\left|\cos \left(\varphi_{0}-\widehat{\varphi}_{0}-\varphi\right)\right|\left(x_{1}-x_{0}\right) \Delta(\mu) .
$$

Moreover, $\Delta$ (illustrated figure 6(a)) depends only on $\ddot{x}_{M}$.

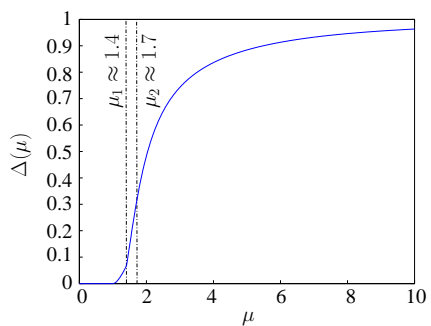

(a)

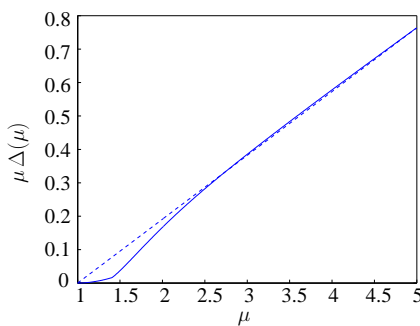

(b)
Fig. 6. Functions $\Delta(\mu)$ on the left and $\mu \Delta(\mu)$ on the right (solid line).

\section{Estimation}

From now on we impose $\widehat{\varphi}_{0}=0$ in (6) and (11), and we propose a way of extracting $\varphi_{0}$ from the relation (11).
Let $\left\{\varphi_{i}\right\}_{1<i<N}$ be a set of $N$ real numbers so that, according to (11), $N$ values $\delta_{i}$ are defined :

$$
\forall i, \delta_{i}=\varepsilon_{i} \cos \left(\varphi_{0}-\varphi_{i}\right) \alpha\left(x_{1}-x_{0}\right) \Delta\left(\mu_{i}\right),
$$

with

$$
\begin{aligned}
\mu_{0} & =\alpha \ddot{x}_{\max } m / f \\
\varepsilon_{i} & =\operatorname{sign}\left(\cos \left(\varphi_{0}-\varphi_{i}\right)\right) \\
\mu_{i} & =\varepsilon_{i} \mu_{0} \cos \left(\varphi_{0}-\varphi_{i}\right) .
\end{aligned}
$$

Let $(i, j) \in\{1, \ldots, N\}^{2}$ and $i \neq j$, according to the previous notations, the following expression is derived :

$$
\frac{\delta_{i}}{\delta_{j}}=\frac{\mu_{i} \Delta\left(\mu_{i}\right)}{\mu_{j} \Delta\left(\mu_{j}\right)} .
$$

Let's note $J_{i j}\left(\mu_{0}, \varphi_{0}\right)$ the function defined by :

$$
J_{i j}\left(\varphi_{0}, \mu_{0}\right)=\left(\delta_{i} \mu_{j} \Delta\left(\mu_{j}\right)-\delta_{j} \mu_{i} \Delta\left(\mu_{i}\right)\right)^{2} .
$$

Keep in mind that $\mu_{i}$, whose definition is given by (13), is a function of both $\varphi_{0}$ and $\mu_{0}$.

Estimating the initial phase comes down to solving the following optimization problem :

$$
\left(\widehat{\varphi}_{0}, \widehat{\mu}_{0}\right)=\arg \min _{\substack{\varphi_{0} \in \mathbb{R} \\ \mu_{0} \in \mathbb{R}^{+}}}\left(\sum_{\substack{i, j \\ j>i}} J_{i j}\left(\varphi_{0}, \mu_{0}\right)\right)
$$

\section{E. Approximated solution}

Even if different iterative methods could be used to solve (15), thanks to an approximation, a less computation demanding solution is derived. The relevancy of the approach is illustrated by experimental results in the next section.

The basic idea developped in this section is to obtain an optimization problem close to (15) but whose solution does not rely on a numerical algorithm. To this end, suppose there exists a constant $\gamma \in \mathbb{R}^{+}$so that $\mu \Delta(\mu)$ is linearized :

$$
\forall \mu \geq 1, \quad \mu \Delta(\mu) \approx \gamma(\mu-1) .
$$

Keep in mind the approximation (16), illustrated figure 6(b) in dotted line, is only valid for $\mu \geq 1$.

The elementary criterion $J_{i j}\left(\varphi_{0}, \mu_{0}\right)$, given by (14), together with (16), is rewritten in $J_{i j}^{*}\left(\varphi_{0}, \mu_{0}\right)$ defined by :

$$
\begin{aligned}
J_{i j}^{*}\left(\varphi_{0}, \mu_{0}\right) & =\gamma^{2}\left(\delta_{i}\left(\mu_{j}-1\right)-\delta_{j}\left(\mu_{i}-1\right)\right)^{2} \\
& =\gamma^{2}\left(\left(\mu_{i} \mu_{j}\right) A_{i j}\left(\begin{array}{c}
\mu_{i} \\
\mu_{j}
\end{array}\right)+b_{i j}^{T}\left(\begin{array}{c}
\mu_{i} \\
\mu_{j}
\end{array}\right)+c_{i j}^{2}\right)
\end{aligned}
$$

with the following notations :

$$
\begin{aligned}
& \text { - } A_{i j}=\left(\begin{array}{cc}
\delta_{j}^{2} & -\delta_{i} \delta_{j} \\
-\delta_{i} \delta_{j} & \delta_{i}^{2}
\end{array}\right) \text {, } \\
& -b_{i j}^{T}=-2\left(\delta_{i}-\delta_{j}\right)\left(\begin{array}{ll}
-\delta_{j} & \delta_{i}
\end{array}\right), \\
& -c_{i j}=\left(\delta_{i}-\delta_{j}\right)^{2} \text {. }
\end{aligned}
$$

The criterion $J\left(\varphi_{0}, \mu_{0}\right)$ to be minimized in (15) is approximated by $J^{*}\left(\varphi_{0}, \mu_{0}\right)$, a quadratic function of the vector $\bar{\mu}=\left(\begin{array}{lll}\mu_{1} & \ldots & \mu_{N}\end{array}\right)^{T}:$

$$
J^{*}\left(\varphi_{0}, \mu_{0}\right)=\gamma^{2}\left(\bar{\mu}^{T} A \bar{\mu}+b^{T} \bar{\mu}+c\right) .
$$


$A$ is a $N \times N$ matrix, $b$ an $N$-dimensional vector and $c$ a scalar. They are defined by :

$$
\begin{array}{l|l}
-\forall(i, j), & \begin{array}{l}
i=j, \\
-\forall>i,
\end{array} \quad A(i, i)=\sum_{j \neq i} \delta_{j}^{2} \\
-\forall i, \quad b(i)=2 \sum_{j \neq i}\left(\delta_{j}\left(\delta_{i}-\delta_{j}\right)\right) \\
-c=\sum_{i, j} c_{i j}
\end{array}
$$

Note that the value of the unknown parameter $\gamma$ can be ommitted to compute the minimum of $J^{*}$.

To make the most of $J^{*}$ being a linear function of $\bar{\mu}$, a coordinates change is necessary. According to the definitions (13), for all $i, \mu_{i}$ is rewritten in cartesian coordinates :

$$
\mu_{i}=\mu_{0}\left(\begin{array}{ll}
\varepsilon_{i} \cos \left(\varphi_{i}\right) & \varepsilon_{i} \sin \left(\varphi_{i}\right)
\end{array}\right)\left(\begin{array}{c}
\cos \left(\varphi_{0}\right) \\
\sin \left(\varphi_{0}\right)
\end{array}\right) .
$$

Then, we define $\theta \in \mathbb{R}^{2}$ and $M$ an $N \times 2$ matrix :

$$
\begin{aligned}
\theta & =\mu_{0}\left(\begin{array}{c}
\cos \left(\varphi_{0}\right) \\
\sin \left(\varphi_{0}\right)
\end{array}\right) \\
M & =\left(\begin{array}{cc}
\varepsilon_{1} \cos \left(\varphi_{1}\right) & \varepsilon_{1} \sin \left(\varphi_{1}\right) \\
\vdots & \vdots \\
\varepsilon_{N} \cos \left(\varphi_{N}\right) & \varepsilon_{N} \sin \left(\varphi_{N}\right)
\end{array}\right)
\end{aligned}
$$

so that $\bar{\mu}=M \theta$ is a linear function of the new optimization variables. Note that $\varphi_{0}$ is the phase of the vector $\theta$ and $\mu_{0}$ its norm.

$J^{*}$ is a function of the optimization variable $\theta$ with :

$$
J^{*}\left(\varphi_{0}(\theta), \mu_{0}(\theta)\right)=\widetilde{J}(\theta)=\theta^{T} M^{T} A M \theta+b^{T} M \theta+c .
$$

Since approximation (16) is valid for $\mu \geq 1$, the minimization of $\widetilde{J}(\theta)$ is subjected to $N$ constraints :

$$
\varepsilon_{i}\left(\cos \left(\varphi_{i}\right) \sin \left(\varphi_{i}\right)\right) \theta \geq 1 .
$$

Let $\widehat{\theta}$ be the solution of this quadratic optimization problem under constraints :

$$
\left\{\begin{array}{l}
\widehat{\theta}=\arg \min _{\theta \in \mathbb{R}^{2}} \widetilde{J}(\theta) \\
\text { Subject to : } \\
\forall i, \quad \varepsilon_{i}\left(\cos \left(\varphi_{i}\right) \quad \sin \left(\varphi_{i}\right)\right) \theta \geq 1
\end{array}\right.
$$

$\widehat{\theta}$ is easily obtained by writting the optimality conditions of Kuhn and Tucker (see [3] for details) and the phase of the vector $\widehat{\theta}$ is $\widehat{\varphi}_{0}$, the estimate of the initial phase.

\section{EXPERIMENTAL RESULTS AND COMPARISON}

\section{A. Implementation}

To solve the approximated optimization problem (19), for each $\varphi_{i}, \delta_{i}$ and $\varepsilon_{i}$ need to be determined from the available measurements.

$M$ is chosen large enough for the periodic orbit to be reached, and, to get rid of transient phenomena, an integer $n_{0}$ is picked up so that $\delta$, defined by (11), is experimentally determined by :

$$
\delta \approx \frac{1}{2 M-n_{0}+1} \sum_{k=n_{0}}^{2 M} \max _{0 \leq \tau \leq T}|x(k T+\tau)-x(k T)| .
$$

Applying relation (20) for each $\varphi_{i}$, we build up the set $\left\{\delta_{i}\right\}_{1<i<N}$.

Let's now consider the system (6) (with $\widehat{\varphi}_{0}=0$ and $\varphi=\varphi_{i}$ ) and recall this system is initially at rest. If $\xi_{1}>\xi_{0}$ (resp. $\xi_{1}<\xi_{0}$ ), then $x(T)$ is larger (resp. smaller) than $x(0)$ if $\cos \left(\varphi_{0}-\varphi_{i}\right)>0$ and, conversely, $x(T)$ is smaller (resp. larger) than $x(0)$ if $\cos \left(\varphi_{0}-\varphi_{i}\right)<0$. Thus, $\varepsilon_{i}$ is easily derived from the following relation :

$$
\varepsilon_{i}=\operatorname{sign}\left(\left(\xi_{1}-\xi_{0}\right)(x(T)-x(0))\right) .
$$

\section{B. Classical method}

We now give a brief description of another initialization method only requiring displacement measurements. For this method, in phase \#1, the current is constant and non-zero and, in phase $\# 2$, the current is zero :

$$
i_{1}=\frac{\widehat{m}}{\widehat{K}_{m}} \ddot{x}_{0}, \quad i_{2}=0 .
$$

$\ddot{x}_{0}$ is constant and homogenous to an acceleration (similarly to (5)). The evolution of the system is then given by :

$$
\ddot{x}=\alpha \sin \left(\frac{2 \pi}{P} d+\varphi_{0}\right) \ddot{x}_{0}-\frac{f}{m} \operatorname{sign}(\dot{x}) .
$$

The corresponding force of the motor generates damped oscillations (because of friction) before an equilibrium position, noted $x_{\infty}$, is reached. The corresponding displacement is $d_{\infty}$ :

$$
\varphi_{0}=\frac{2 \pi}{P} x_{\infty}-\frac{2 \pi}{P} d_{\infty} \bmod 2 \pi .
$$

If friction is not too significant, the following relation holds :

$$
\exists k_{\infty} \in \mathbb{Z}, \quad x_{\infty} \approx \widehat{x}_{\infty}=\frac{P}{2}+k_{\infty} P .
$$

With no additionnal information, a possible estimate for $\varphi_{0}$ is :

$$
\begin{aligned}
\widehat{\varphi}_{0} & =\frac{2 \pi}{P} \widehat{x}_{\infty}-\frac{2 \pi}{P} d_{\infty} \bmod 2 \pi \\
& =\pi-\frac{2 \pi}{P} d_{\infty} \bmod 2 \pi .
\end{aligned}
$$

Actually, when the friction $f$ is significant, the previous estimate is highly biased and it can be shown that the estimation error is related to the parameter $\mu^{\prime}=\alpha m \ddot{x}_{0} / f$. The smaller $\mu^{\prime}$, the worse the estimation.

\section{Results}

We illustrate the results of our method in comparison with the classical procedure. To this end, let's consider two linear motors whose parameters are unknown but the friction is known to be more important for the second motor, even though this information is not used by the initialization algorithms. 
According to the previous notations, the first motor is defined by $\alpha_{1}, m_{1}, f_{1}$ and the second by $\alpha_{2}, m_{2}, f_{2}$.

To compare the methods, we impose $\mu_{0}=\mu^{\prime}$ ( $\mu_{0}$ is defined by (13)) which turns out to be equivalent to $\ddot{x}_{0}=\ddot{x}_{\max }$ $\left(\ddot{x}_{\max }\right.$ is given by (9)). We estimate the average estimation error $\widehat{\varphi}_{0}-\varphi_{0}$ when $\varphi_{0}$ is varied from 0 to $2 \pi$.

Let's consider the first linear motor to experimentally check out that the precision of the classical method worsens as $\mu^{\prime}=\alpha_{1} m_{1} \ddot{x}_{0} / f_{1}$ decreases while our solution does not depend upon $\mu_{0}=\alpha_{1} m_{1} \ddot{x}_{\max } / f_{1}$. To this end, we consider the following experimental conditions :

$$
\begin{aligned}
& -\ddot{x}_{0}=\ddot{x}_{\text {max }}=1000 \mathrm{~mm} / \mathrm{s}^{2} \text { (figure 7(a)), } \\
& -\ddot{x}_{0}=\ddot{x}_{\text {max }}=500 \mathrm{~mm} / \mathrm{s}^{2} \text { (figure 7(b)). }
\end{aligned}
$$

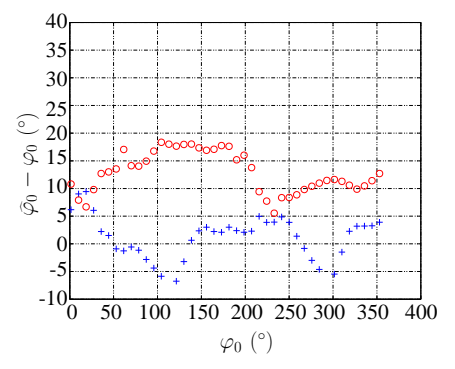

(a) $\ddot{x}_{0}=\ddot{x}_{\max }=1000 \mathrm{~mm} / \mathrm{s}^{2}$

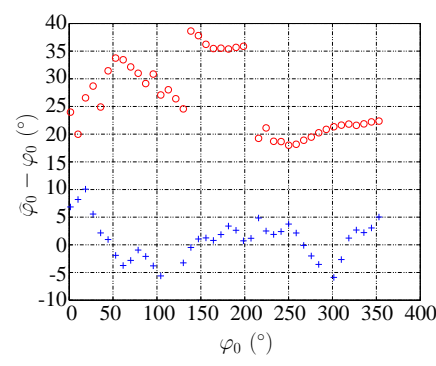

(b) $\ddot{x}_{0}=\ddot{x}_{\max }=500 \mathrm{~mm} / \mathrm{s}^{2}$
Fig. 7. Average estimation error $\widehat{\varphi}_{0}-\varphi_{0}$ versus initial phase $\varphi_{0}$. Our method (19), solid line, and the classical method (22), dotted line.

As expected, the method of the paper offers the same accuracy when $\mu_{0}$ is varied. For this motor, the precision on the initial phase is better than $10^{\circ}$, which confers an efficiency of $98 \%$ to the motor. For high accelerations (figure 7(a)), the classical method gives slightly less accurate results but generates erratic and large magnitude displacements. As expected, when $\mu^{\prime}$ decreases (figure $7(\mathrm{~b})$ ), this method exhibits a precision of $30^{\circ}$ and a corresponding efficiency of $87 \%$ while our procedure still offers the same $98 \%$ efficiency. For figure 7 and the motor with little friction, the precision of our method is constant and independent of the motor parameters $\left(\mu_{0}\right)$ and gives a substantially better energetic efficiency to the motor.

Let's now move on to the second motor which exhibits important friction so that the reference acceleration has to be larger $\left(\ddot{x}_{\max }=\ddot{x}_{0}=4000 \mathrm{~mm} / \mathrm{s}^{2}\right)$. The average estimation error is given for both the methods on figure 8 . Our method

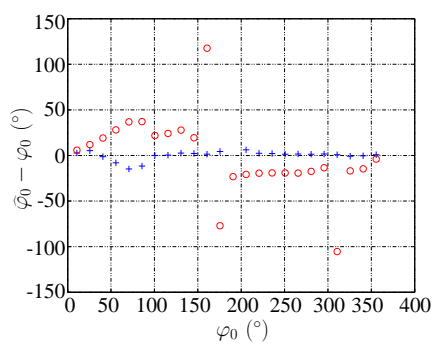

Fig. 8. Average estimation error $\widehat{\varphi}_{0}-\varphi_{0}$ versus initial phase $\varphi_{0}$. Our method (19), line, and the classical method (22), dotted line.

is very robust to dry friction since it still achieves the same precision (approximately $10^{\circ}$ ). On the contrary, the classical does not perform that well.

The method of the paper turns out to be independent of $\mu_{0}$ which means insensibility to friction, load and motor gain. Whatever values these parameters may take, for the considered motors, our method outperforms the classical procedure and a precision of $10^{\circ}$ is achieved.

\section{CONClusion}

A new method to determine the initial phase for synchronous motors has been proposed. No information about the motor internal functioning is required and the algorithm only has to be fed with relative position measurements from incremental coders. For high precision applications, no additionnal hardware is needed which allows to get rid of Hall effect sensors. Our solution exhibits better results than the classical method though implemented in the same conditions. Despite friction, poorly identified motor gain or unknown motor load, our method features precise initial phase estimation which guarantees optimal energetic use of synchronous motors.

\section{REFERENCES}

[1] J. Boichot, E. Delaleau, N. V. Diep, J. Lévine and E. Parzy, « Modelling And Control Of A High-Precision Positioning System Actuated By A Linear Synchronous Motor», Proc. IFAC, Beijing, China, 1999.

[2] J. Boichot, E. Delaleau, N. V. Diep, J. Lévine and E. Parzy, « Modelling and Control Of A Two D.O.F. High-Precision Positioning System », Proc. ECC, Karlsruhe, Allemagne, 1999.

[3] J.C. Culioli, Introduction à l'Optimisation, Ellipses, 1994.

[4] R. Dhaouadi, N. Mohan and L. Norum, « Design And Implementation Of An Extended Kalman Filter For The State Estimation Of A Permanent Magnet Synchronous Motor », IEEE Trans. Power Electronics, vol. 6, pp. 491-497, May 1991.

[5] A.F. Filippov, Differential Equations with Discontinuous Righthand Sides, Kluwer Academic Publishers, 1988.

[6] J. Guckenheimer and P.Holmes, Nonlinear Oscillations, Dynamical Systems, and Bifurcations of Vector Fields, Springer Verlag, 1986.

[7] A. B. Kulkarni and M. Ehsani, « A Novel Position Sensor Elimination Technique For The Interior Permanent Magnet Synchronous Motor Drive », IEEE Trans. Industry Applications, vol. 28, pp. 144-150, Jan./Feb. 1992.

[8] K. W. Lim, K. S. Low and M. F. Rahman, « Observers For Sensorless Control Of Permanent Magnet Synchronous Motor Drive », Proc. IFAC, Sydney, Australia, vol. 2, pp. 431-434, 1993.

[9] T. Noguchi, K. Yamada, S. Kondo and I. Takahashi, "Initial Rotor Position Estimation Method Of Sensorless PM Motor With No Sensitivity To Armature Resistance », IEEE Trans. Industrial Electronics, vol. 45, pp. 118-125, Feb. 1998.

[10] S. Ogasawara and H. Agaki, « An Approach To Real Time Position Estimation At Zero And Low Speed For A PM Motor Based On Saliency », IEEE Industry Applications Society Annual Meeting, pp. 2935, Oct 6-10 1996.

[11] S. Ostlund and M. Brokemper, "Sensorless Rotor-Position Detection From Zero To Rated Speed For An Integrated PM Synchronous Motor Drive », IEEE Trans. Industry Applications, vol 32, pp. 1158-1165, Sept./Oct. 1996.

[12] P. B. Schmidt, M. L. Gasperi, G. Ray, A. H. Wijenayake, « Initial Rotor Angle Detection Of A Non-Salient Pole Permanent Magnet Synchronous Machine », IEEE Industry Applications Society Annual Meeting, pp. 459463, 1997. 